\title{
Estudio De Tiempos Y Rendimientos En La Transformacion De Trozas De Madera Pinus Radiata D. Don (Pino) En Tablas Para La Elaboracion De Pallets
}

\author{
Ing. Eduardo Cevallos (Ing. Agrónomo), \\ Ximena Lara (Ing. Forestal), \\ Janneth Morocho (Doctora), \\ Martha Dávalos (Doctora), \\ Eduardo Salazar (Ing. Forestal), \\ Sulema Lara (Ing. Ecot.)
}

Escuela Superior Politécnica De Chimborazo, Ecuador

doi: 10.19044/esj.2017.v13n10p80 URL:http://dx.doi.org/10.19044/esj.2017.v13n10p80

\begin{abstract}
This present study gives an estimation of the time and efficiency in the transformation of pine logs into boards for the manufacturing of pallets in the industry, Haro Madera. Here, the percentage of the wood used and the percentage of sawdust were determined. This was with a yield of $34.936 \%$ in the processing of the logs, $43.29 \%$ into square posts, and $15.08 \%$ in the direct transformation of the logs into boards. In order to determine the factors that affect the production of pine lumber, the wood was classified by its length, diameter, and shape where it was possible to determine its influence in the production into boards. This, therefore, proves that the qualitative factors are influential in the elaboration of the boards to construct pallets, in terms of time. Also, it was possible to determine that for the manufacture of an American type pallet, it took 10.24 minutes, while the European type pallet took 13.9 minutes. To determine the output per machine, a sampling was used. Here, specific observations were made, dividing working time and non-working time. Wood utilization percentage was low, the production line must be improved, and the logs from different plantations should be classified according to their diameter and shape.
\end{abstract}

Keywords: Pallets, efficiency, pine logs, pine boards 


\section{Resumen}

El presente estudio comprende en estimar los tiempos y rendimientos en la transformación de las trozas de pino en tablas para la elaboración de pallets en la industria Haro Madera, para lo cual se estableció el porcentaje de madera utilizada y la madera conocida como merma producto del aserrío de las trozas de pino, siendo su rendimiento de 34,936 \% en la elaboración de troza a bloque y de 43,29 \% de bloque a tabla, y de 15,08 \% en la transformación directa de troza a tabla, para determinar los factores que inciden en el rendimiento de la madera de pino, se realizó la clasificación de la madera por su longitud, diámetro y forma en donde se pudo determinar su influencia, comprobándose de ésta manera que los factores cualitativos influyen en la elaboración de las tablas para elaborar el pallet, en cuanto a los tiempos se pudo determinar que para la fabricación del pallet tipo americano se obtuvo 10,24 minutos y para el pallet tipo europeo se obtuvo 13,09 minutos. Para determinar el rendimiento por máquina, se utilizó un muestreo, donde se hicieron observaciones puntuales, dividiendo al tiempo en trabajado y no trabajado. El porcentaje de aprovechamiento de la madera es baja, se debe mejorar la línea de producción, clasificar las trozas provenientes de las distintas plantaciones por su diámetro y forma.

Palabras clave: Pallets, eficiencia, trozas de pino, tablones de pino

\section{Introducción}

La globalización económica ha conducido a una mayor competencia entre las empresas forestales, lo que ha incrementado el interés por tomar mejores decisiones, con el fin de aprovechar al máximo la materia prima disponible y optimizar el proceso de producción y, de forma paralela, reducir costos; además de aumentar la calidad (Souza, 2007). El aprovechamiento racional de los recursos forestales desempeña un rol importante en el desarrollo económico y social del país; por ello, disminuir la pérdida de materia prima, mejorar la calidad de los productos, así como optimizar el uso de la mano de obra y de los equipos, son tareas pendientes y en continuo desarrollo en el sector productivo (Aguilera et al., 2005).

La fabricación de madera aserrada está influenciada, mayormente, por el precio de la materia prima en rollo, que a su vez, depende de los costos de manejo, extracción y transporte, por lo que lo que su venta al consumidor final incluye los costos de transformación y manejo a través de toda la cadena productiva (Semarnat, 2002). Infor (2009) señala que en las actividades forestales, los factores de conversión que relacionan la cantidad de madera en trozas por unidad de producto final se emplean como herramientas de apoyo para el manejo forestal y al sector industrial; al mismo tiempo, son fundamentales para la elaboración de estadísticas 
sectoriales, estudios, análisis, proyecciones y aproximaciones de producción en las diversas ramas de la actividad forestal como la silvicultura, específicamente en cálculos de biomasa, captura de carbón; y podrían utilizarse por las empresas para valorarlas ventas y estimar precios por unidad de madera aserrada La industria maderera en el Ecuador es una de las principales actividades del país, siendo una fuente directa e indirecta de ingresos; y es la encargada de dar valor agregado a los productos forestales y de esta manera contribuir a la conservación de los recursos boscosos mediante su aprovechamiento adecuado tanto en la construcción como materia prima para mercados nacionales e internacionales.

En la provincia de Chimborazo las industrias madereras se han concentrado en su mayoría en las zonas urbanas, las mismas que albergan la mayor cantidad de depósitos, aserraderos y otras empresas destinadas a la elaboración de productos provenientes de la madera.

En la parroquia Calpi donde se encuentra ubicado el aserradero Haro Madera, se realizó el análisis de tiempos y rendimientos en la transformación de trozas de madera Pinus radiata D. DON (pino) en tablas para la elaboración de pallets. En éste estudio verificamos su eficacia en cuanto a la relación entre el volumen de madera en rollo y el volumen resultante en productos aserrado, la misma que en promedio es de apenas el $13.65 \%$, que existe una correlación fuerte negativa de 0,95 entre la variable diámetro medio y la variable porcentaje de rendimiento. Por otro lado el tiempo promedio de elaboración de pallets americanos y europeos es de 10.24 y 13.09 minutos respectivamente. Además debido a la impericia de la mano de obra se concluye que por cada troza se obtiene el mismo volumen de madera aserrada, ocasionando desperdicio y pérdida para la empresa.

\section{Texto principal}

Para realizar este estudio se aplicó el análisis de tiempos y rendimientos así como investigación bibliográfica y de campo la misma que se detalla en las siguientes fases:

\section{Clasificación de las trozas por su diámetro longitud y volumen}

La clasificación de las trozas de pino que llegan al aserradero se lo hizo en base a su diámetro, clasificándolas en trozas de similares dimensiones en diámetro, cuyos promedios en general llegaron a $32 \mathrm{~cm} \mathrm{y}$ longitud de 2,52 $\mathrm{m}$.

El número de trozas registradas por viaje fueron de 20 trozas, las mismas que fueron enumeradas en un extremo con la finalidad de evitar que se mezclen con otras trozas que ingresan en el patio de almacenamiento y de esta manera dar un seguimiento a cada una de ellas al ingresar a la sierra para la elaboración de tablas. 
La cubicación de las trozas se las realizó mediante el empleo de la formula Smalian, donde se promedian los diámetros mayor y menor; para la determinación del volumen del bloque se utilizó las medidas de largo ancho y profundidad.

\section{Determinación y rendimiento de maquinaria de aserrío en la transformación de trozas}

Una vez seleccionadas las trozas se registraron los datos referentes a: diámetros menor y mayor, longitud y tiempo. Las trozas aserradas permitieron el registro de las dimensiones del prisma rectangular; finalmente se registraron las dimensiones de las tablas provenientes del mismo, que fueron utilizadas para la elaboración de los pallets. La información obtenida en los procesos descritos anteriormente permitieron calcular el rendimiento de las trozas y el tiempo promedio de elaboración de los pallets.

Para el armado de pallets tipo americano y tipo europeo se tomó el tiempo en las operaciones realizadas por las diferentes maquinas: Wood mizer, sierra de cinta, machimbradora, cepilladora, trozeadora, multisierras.

\section{Resultados}

Análisis del rendimiento en la transformación de la madera: TrozaBloque y Bloque-Tabla

Para determinar el rendimiento de la madera en su transformación se registraron las mediciones descritas anteriormente lo que permitió obtener los siguientes resultados:

Tabla 1. Volumen y Rendimiento en la transformación de la madera

\begin{tabular}{|c|c|c|c|c|c|c|c|c|c|}
\hline \multirow[b]{2}{*}{$\mathrm{N}^{\circ}$ Trozas } & Vol. & $\mathrm{N}^{\circ}$ & Vol. & $\%$ & $\%$ & $\mathrm{~N}^{\circ}$ & Vol. & $\%$ & $\%$ \\
\hline & $\begin{array}{l}\text { Trozas } \\
\text { (m3) }\end{array}$ & Bloques & $\begin{array}{c}\text { Bloques } \\
\left(\mathrm{m}^{3}\right)\end{array}$ & $\begin{array}{l}\text { Rendimiento } \\
\text { Troza-Bloque }\end{array}$ & Desperdicios & Tablas/bloque & Tablas & \begin{tabular}{|c|} 
Rendimiento \\
Bloque-Tablas
\end{tabular} & Desperdicios \\
\hline 1 & 0,3342 & 1 & 0,1334 & 39,90 & 60,1010 & 20 & 0,0572 & 42,89 & 57,1054 \\
\hline 2 & \begin{tabular}{|l|}
0,2752 \\
\end{tabular} & 2 & 0,1360 & 49,41 & 50,5903 & 20 & 0,0572 & 42,07 & 57,9334 \\
\hline 3 & 0,4440 & 3 & 0,1323 & 29,80 & 70,2032 & 20 & 0,0572 & 43,24 & 56,7649 \\
\hline 4 & 0,3402 & 4 & 0,1307 & 38,43 & 61,5748 & 20 & 0,0572 & 43,76 & 56,2440 \\
\hline 5 & 0,4162 & 5 & 0,1318 & 31,66 & 68,3413 & 20 & 0,0572 & 43,41 & 56,5927 \\
\hline 6 & 0,5778 & 6 & 0,1208 & 20,90 & 79,1003 & 20 & 0,0572 & 47,37 & 52,6294 \\
\hline 7 & 0,1851 & 7 & 0,1339 & 72,34 & 27,6593 & 20 & 0,0572 & 42,73 & 57,2736 \\
\hline 8 & 0,5125 & 8 & 0,1360 & 26,53 & 73,4660 & 20 & 0,0572 & 42,07 & 57,9334 \\
\hline 9 & 0,2542 & 9 & 0,1307 & 51,43 & 48,5744 & 20 & 0,0572 & 43,76 & 56,2440 \\
\hline
\end{tabular}




\begin{tabular}{|c|c|c|c|c|c|c|c|c|c|}
\hline 10 & 0,5739 & 10 & 0,1402 & 24,43 & 75,5738 & 20 & 0,0572 & 40,81 & 59,1939 \\
\hline 11 & 0,4575 & 11 & 0,1397 & 30,52 & 69,4787 & 20 & 0,0572 & 40,96 & 59,0405 \\
\hline 12 & 0,6320 & 12 & 0,1355 & 21,43 & 78,5679 & 20 & 0,0572 & 42,23 & 57,7704 \\
\hline 13 & 0,4196 & 13 & 0,1328 & 31,66 & 68,3413 & 20 & 0,0572 & 43,06 & 56,9358 \\
\hline 14 & 0,2779 & 14 & 0,1334 & 47,98 & 52,0209 & 20 & 0,0572 & 42,89 & 57,1054 \\
\hline 15 & 0,4552 & 15 & 0,1229 & 26,99 & 73,0118 & 20 & 0,0572 & 46,56 & 53,4392 \\
\hline 16 & 0,4941 & 16 & 0,1334 & 26,99 & 73,0118 & 20 & 0,0572 & 42,89 & 57,1054 \\
\hline 17 & 0,3420 & 17 & 0,1323 & 38,69 & 61,3130 & 20 & 0,0572 & 43,24 & 56,7649 \\
\hline 18 & 0,4422 & 18 & 0,1318 & 29,80 & 70,2032 & 20 & 0,0572 & 43,41 & 56,5927 \\
\hline 19 & 0,3402 & 19 & 0,1307 & 38,43 & 61,5748 & 20 & 0,0572 & 43,76 & 56,2440 \\
\hline 20 & 0,5977 & 20 & 0,1281 & 21,43 & 78,5679 & 20 & 0,0572 & 44,65 & 55,3474 \\
\hline TOTAL & 8,3717 & & 2,6460 & 698,724 & 1301,2756 & & 1,1440 & 865,740 & 1134,260 \\
\hline PROMEDIO & 0,419 & & 0,1323 & 34,94 & 65,064 & & 0,0572 & 43,29 & 56,71 \\
\hline
\end{tabular}

Grafico 1. Rendimiento en la transformación de la madera

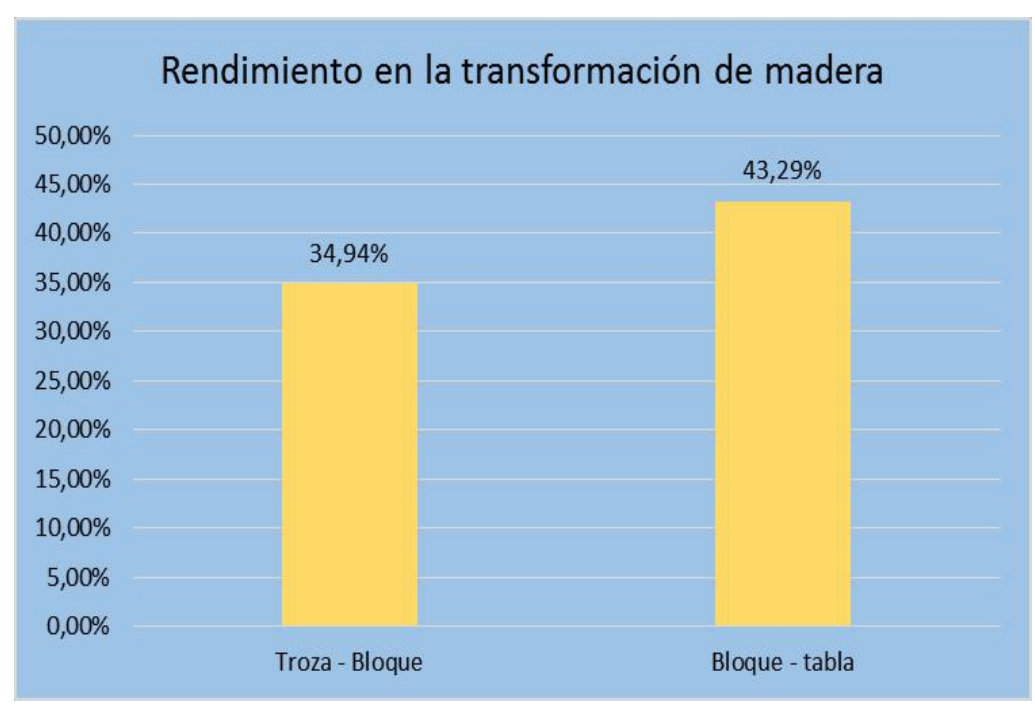

En la Tabla 1 y grafico 1 se puede apreciar un volumen promedio de $0,419 \mathrm{~m} 3$, con un volumen en bloque de $0,1323 \mathrm{~m} 3$ y el volumen de la tabla de $0,0572 \mathrm{~m} 3$. El rendimiento obtenido en la especie Pinus radiata D. Don durante las fases de transformación de troza a bloque, reportó el 34,936 \% y de bloque a tablas con el 43,29\%. 


\section{Análisis de regresión lineal para las variables de aserrío}

Tabla 2. Regresión lineal para diámetro medio (m3) y rendimiento de madera aserrada

\begin{tabular}{|c|c|c|c|c|c|}
\hline $\mathrm{N}^{\circ}$. Trozas & \begin{tabular}{|c|}
$\begin{array}{c}\text { Diámetro Medio } \\
(\mathrm{m})\end{array}$ \\
\end{tabular} & $\begin{array}{l}\text { Rendimiento \% } \\
\text { (trozas - tablas) }\end{array}$ & $x^{2}$ & $y^{2}$ & $x y$ \\
\hline 1 & 0,29 & 17,11 & 0,0838 & 292,610 & 4,9521 \\
\hline 2 & 0,26 & 20,77 & 0,0677 & 431,574 & 5,4045 \\
\hline 3 & 0,34 & 12,88 & 0,1122 & 165,794 & 4,3135 \\
\hline 4 & 0,30 & 16,80 & 0,0870 & 282,399 & 4,9574 \\
\hline 5 & 0,33 & 13,74 & 0,1056 & 188,655 & 4,4639 \\
\hline 6 & 0,40 & 9,90 & 0,1600 & 97,916 & 3,9581 \\
\hline 7 & 0,22 & 30,89 & 0,0462 & 954,368 & 6,6420 \\
\hline 8 & 0,36 & 11,16 & 0,1260 & 124,462 & 3,9605 \\
\hline 9 & 0,26 & 22,49 & 0,0650 & 505,814 & 5,7350 \\
\hline 10 & 0,37 & 9,96 & 0,1369 & 99,248 & 3,6861 \\
\hline 11 & 0,33 & 12,50 & 0,1096 & 156,126 & 4,1359 \\
\hline 12 & 0,40 & 9,05 & 0,1560 & 81,832 & 3,5732 \\
\hline 13 & 0,33 & 13,63 & 0,1056 & 185,685 & 4,4286 \\
\hline 14 & 0,26 & 20,57 & 0,0697 & 423,123 & 5,4305 \\
\hline 15 & 0,35 & 12,56 & 0,1239 & 157,742 & 4,4210 \\
\hline 16 & 0,35 & 11,57 & 0,1239 & 133,879 & 4,0729 \\
\hline 17 & 0,29 & 16,72 & 0,0864 & 279,486 & 4,9150 \\
\hline 18 & 0,34 & 12,93 & 0,1122 & 167,118 & 4,3307 \\
\hline 19 & 0,30 & 16,80 & 0,0870 & 282,399 & 4,9574 \\
\hline 20 & 0,40 & 9,57 & 0,1560 & 91,492 & 3,7782 \\
\hline Sumatoria & 6,44 & 301,58 & 2,12 & 5101,72 & 92,12 \\
\hline Promedio & 0,3219 & 15,08 & & & \\
\hline Varianza & 0,0026 & 29,17 & & & \\
\hline $\begin{array}{l}\text { Desviación } \\
\text { Estándar }\end{array}$ & 0,0507 & 5,4013 & & & \\
\hline $\begin{array}{c}\text { Coeficiente de } \\
\text { Varianza } \\
\end{array}$ & 0,7979 & 35,8206 & & & \\
\hline Err Est & 0,0113 & 1,2078 & & & \\
\hline B & $-101,5568$ & & & & \\
\hline A & 47,7682 & & & & \\
\hline$Y$ & $\begin{array}{c}47,7682- \\
101,5568 \mathrm{x}\end{array}$ & & & & \\
\hline
\end{tabular}


Gráfico 2. Relación entre diámetro medio y rendimiento

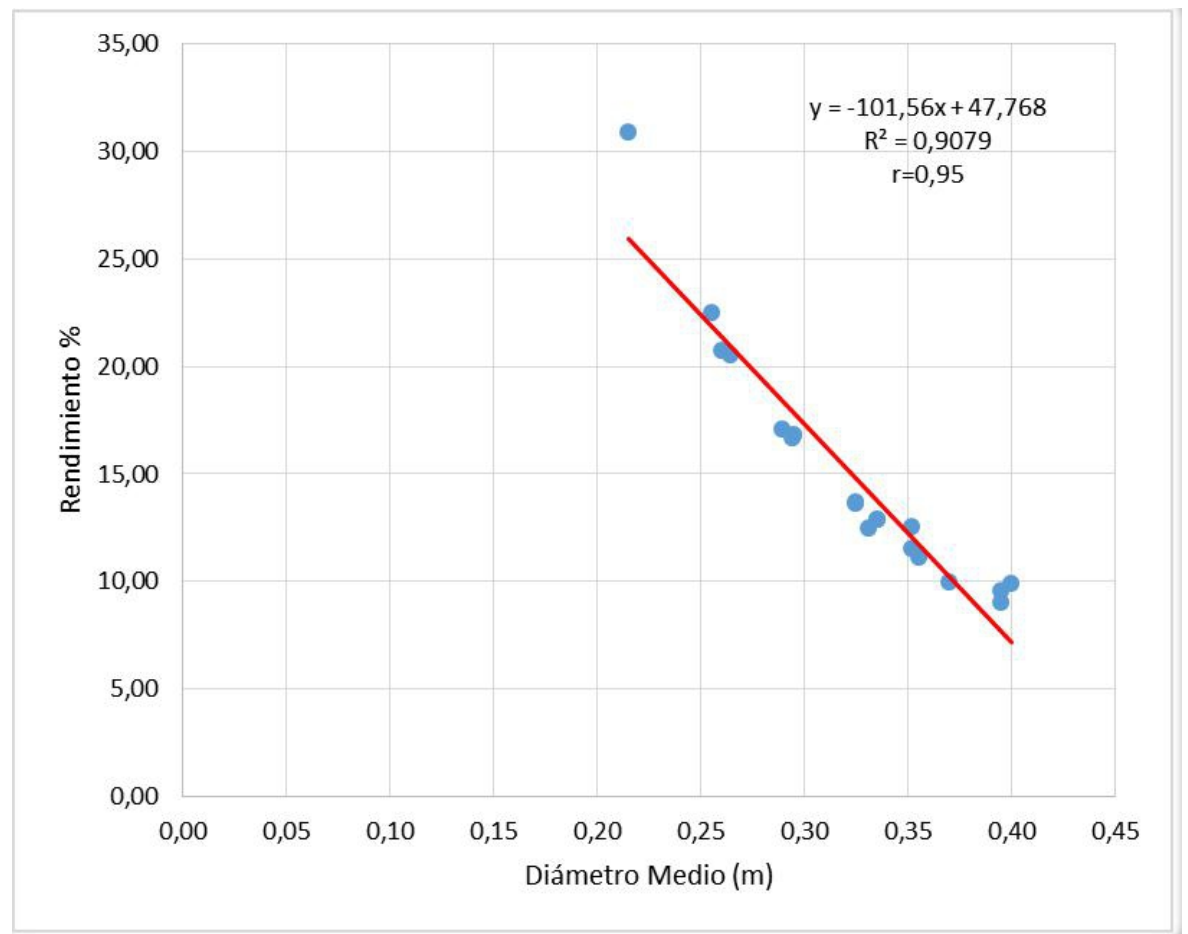

Según la información obtenida en la tabla 2 y el grafico 2 tenemos que la variable " $\mathrm{X}$ " que representa el diámetro medio que tiene un promedio de 0,32 m con desviación estándar $0,0507 \mathrm{~m}$. En cuanto a la variable "Y" que representa el rendimiento troza - tabla tiene un promedio de $15,08 \%$, con desviación estándar es de 5,4013 \%. Existe una alta correlación entre las dos variables siendo $r=0,95.3 .3$. Tiempos de las maquinarias en el aserrado de madera

Tabla 3. Tiempos de maquinarias en el aserrado de madera

\begin{tabular}{|c|c|c|c|c|c|}
\hline Maquinaria & Promedio & $\begin{array}{c}1 \text { pallets } \\
\text { americano }\end{array}$ & $\begin{array}{c}\text { \% de pallets } \\
\text { americano }\end{array}$ & $\begin{array}{c}1 \text { pallets } \\
\text { europeo }\end{array}$ & $\begin{array}{c}\% \text { pallets } \\
\text { Europeo }\end{array}$ \\
\hline Wood Mizer & 9,488 & 5,22 & 50,94 & 6,64 & 50,72 \\
\hline Sierra de Cintas & 0,143 & 0,08 & 0,77 & 0,10 & 0,76 \\
\hline Machimbradora & 0,036 & 0,40 & 3,87 & 0,50 & 3,85 \\
\hline Cepilladora & 0,0465 & 0,51 & 4,99 & 0,65 & 4,97 \\
\hline Trozeadora & 0,1660 & 1,83 & 17,83 & 2,32 & 17,75 \\
\hline Multisierras & 0,0630 & 0,69 & 6,77 & 0,88 & 6,74 \\
\hline Armado Pallets Europeos & 1,9915 & & & 1,992 & 15,21 \\
\hline $\begin{array}{c}\text { Armado Pallets } \\
\text { Americanos }\end{array}$ & 1,52 & 1,52 & 14,84 & & \\
\hline Total & 13,45 & 10,24 & & 13,09 & \\
\hline
\end{tabular}


Grafico 3. Porcentajes de tiempo de las maquinas en la elaboración de Pallets Americano

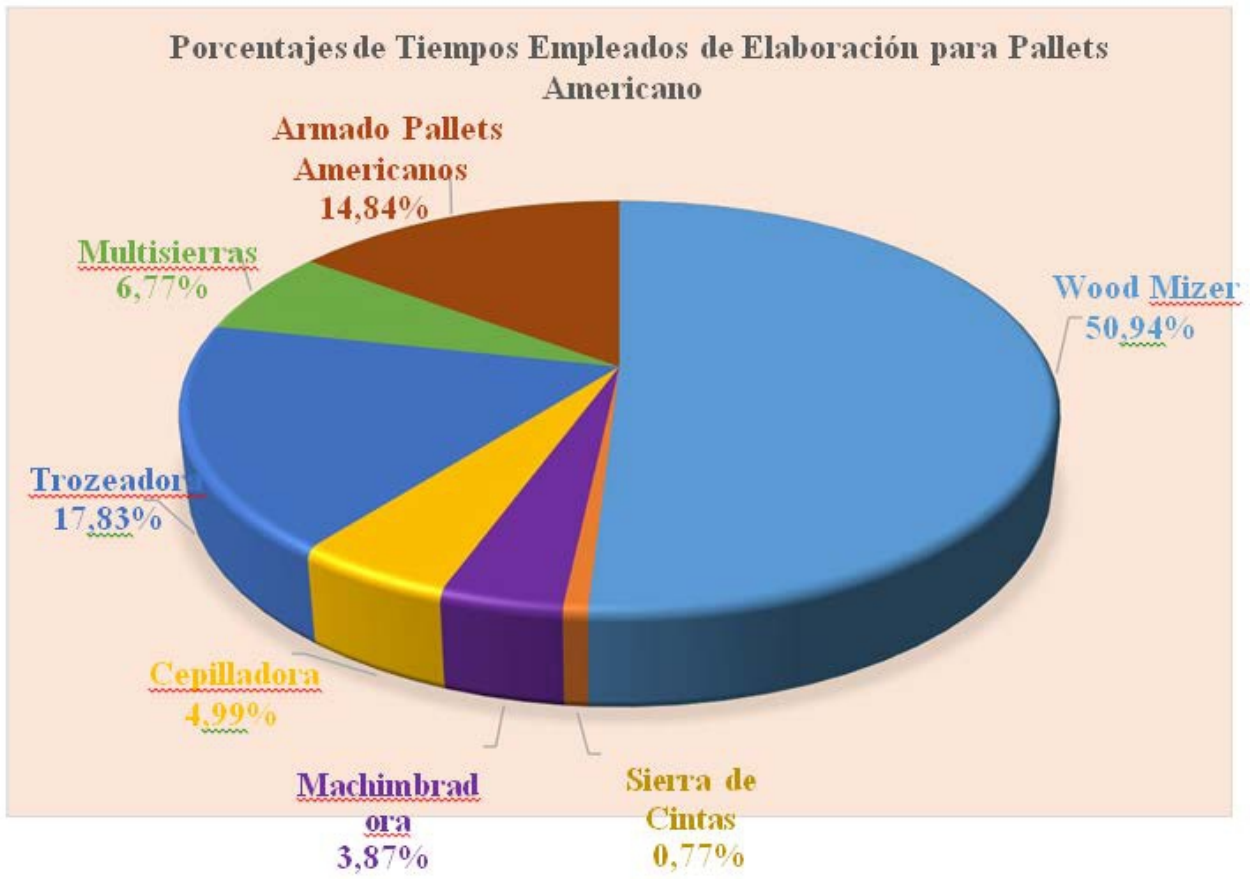

Grafico 4. Porcentajes de tiempo empleados de las maquinas en la elaboración de Pallets Porcentajes de Tiempos Empleados de Elaboración para Pallets

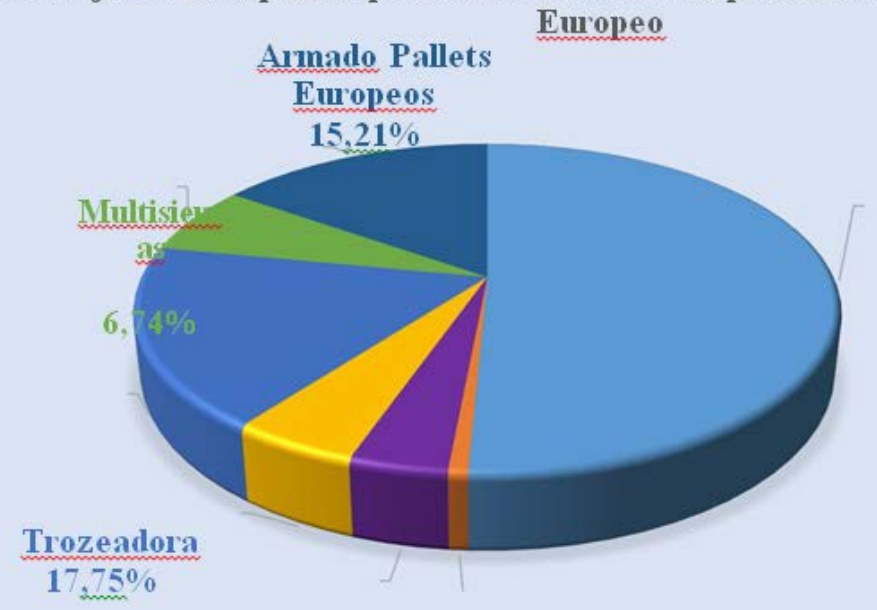

\section{Cepilladora}

\section{Machimbradora Sierra de \\ Cintas} $3,85 \%$

$$
0,76 \%
$$


La tabla 3, grafico 3 y 4, hace referencia al promedio de tiempos de cada maquinaria para el proceso de elaboración de los pallets americano de 10,24 minutos y para un pallets europeos con 13,09 minutos.

\section{Conclusion}

La mayoría de trozas, que llegan al aserradero poseen mismas características de forma y sanidad.

El mayor rendimiento de la madera se la obtiene al trasformar de bloque de madera a tabla con un $43,29 \%$, con un desperdicio de $56.71 \%$.

El menor rendimiento de madera se da en la transformación de troza a bloque con apenas 34,94\% y un desperdicio de 65,06\% debido a que las trozas deben tener una misma dimensión.

El volumen promedio de las trozas se $0,419 \mathrm{~m}^{3}$ con un tiempo promedio de 9,63 minutos en la trasformación de troza a tablas.

$\square$ En el proceso de transformación de la madera existe un desprecio de troza - bloque con un $65,064 \%$ y de bloque a troza un $56,71 \%$, pero no es una perdida para la empresa ya que todos los desprecios lo venden a empresas demandantes.

\section{References:}

1. AGUILERA, A, INSUNZA, L. ALZAMORA, R \& TAPIA, L. (2005). Evaluación del costo de producción para faenas de aserrío portátil. Bosque 26(2):107-114.

2. DENIG, J. (1990). Control de la calidad en aserraderos de pino del sur. North Carolina, US, Cooperative Extension Service. 47 p.

3. EGAS, AF. (1998). Consideraciones para elevar los rendimientos en aserraderos con sierras de banda. Tesis Dr. CC Forestales. Cuba, Universidad de Pinar del Río. 100p.

4. INFOR (Instituto Forestal) (2009). Actualización de factores de conversión en el sector forestal de Chile. Primera etapa. Gobierno de Chile. Ministerio de Agricultura. Santiago de Chile, Chile. 35 p.

5. RODRÍGUEZ, BARRERA, CUERVO, \& HERNÁNDEZ (2010). Manual de buenas prácticas en aserraderos de comunidades forestales. México. Consultado en línea en: http://eramx.org/biblio/aserraderos4.pdf.

6. SEGEPLAN (Secretaria General de Planificación, GT) (1994). Taller de trabajo sobre conceptos y definiciones fundamentales de geografía; temática para planificación regional: memoria. Guatemala, Guatemala. 31 p.

7. SEMARNAT (Secretaría del Medio Ambiente y Recursos Naturales) (2002). Texto guía forestal. Dirección General Forestal. México. D. F., México. 159 p. 
8. SOUZA, N. (2007). Um sistema de optimização aplicada o desdobro de madeira. Dissertação (Mestrado). Curso de Pós- Graduação em Engenharia Florestal, UFPR. Curitiba, Paraná, Brasil. 126 p.

9. TOLEDO, E. (2002). Proyecto de desarrollo industrial y comercial de maderas Latifoliadas poco conocidas sobre base sostenible en Guatemala, fase 1. Guatemala, Plan Acción Forestal de Guatemala. $36 \mathrm{p}$.

10. TOLOSANA, E., AMBROSIO, T., \& VIGNOTE, S. (2002). Rendimientos, costes y efectos ambientales de las claras mecanizadas sobre repoblaciones de Pinus sylvestris L. en España. Invest. Agr. Sist. Recur. For. 11(1):39-65.

11. URRA, G. (1999). Estudio de tiempo, rendimiento y costo para los equipos de madereo mecanizado: logger's dream y skidder. (Tesis de Grado. Ingeniero Forestal). Universidad de Talca. Facultad de Ciencias Forestales. Talca - Chile. 68 pp.

12. VERGARA, M. (2000). Evaluación del rendimiento de torres de madereo en faenas a tala rasa. (Tesis de Grado. Ingeniero Forestal). Universidad de Talca. Facultad de Ciencias Forestales. Talca - Chile. $75 \mathrm{pp}$.

13. WOOD-MAIZER, CL. (2006). Especificaciones técnicas del aserradero LT-40 HD y SD, agencia de Chile (en línea). Consultado 27 ago 2006. Disponible en http://www.woodmizer.cl/sawmills/sawprofe-lt40hydraulic.htm.

14. ZABALA, D. (1991). Manual para el establecimiento de un sistema de control de la variación de refuerzos en madera aserrada. Chapingo, México, Universidad Autónoma de Chapingo. 49 p. (Serie de Apoyo Académico no.43). 